\title{
Factors Affecting the Germination of Spores of Clostridium bifermentans
}

\author{
By P. A. GIBBS \\ The Wellcome Research Laboratories (Biological Division), Beckenham, Kent
}

(Received 21 April 1961)

\section{SUMMARY}

The germination of spores of Clostridium bifermentans in media based on acid hydrolysates of casein has been studied. Germination of spores was estimated by observing the decrease in turbidity of a spore suspension and confirmed by observation with the phase contrast microscope and by the increase in permeability to stains. The optimum conditions for germination of spores suspended in phosphate buffer were incubation at $37^{\circ}$ within the range $\mathrm{pH} 6 \cdot 0-7 \cdot 8$ following heat shock at $85^{\circ}$ for $10 \mathrm{~min}$. Anaerobic conditions were not necessary for germination, and addition of mercaptoacetate decreased the rate of germination. The minimum requirements of compounds needed for the germination of spores of $C$. bifermentans was the presence of $\mathrm{L}-\alpha$-alanine, L-phenylalanine and lactate. L-phenylalanine could be replaced by L-leucine but the rate of germination was slower. L- $\alpha$-Alanine was essential for germination and was not replaced by any other amino acid examined.

\section{INTRODUCTION}

Though there is now a considerable volume of published work on the optimum conditions for and specific stimulants of germination of spores of the aerobic genus Bacillus, there is comparatively little information of this type about the spores of the anaerobic genus Clostridium. Such information as is available appears to be largely confined to those obligate anaerobes which have economic and pathological importance. Much of the work is also restricted in that the major emphasis has been on the destruction of these spores or the prevention of germination. Clostridium bifermentans, a non-pathogenic obligate anaerobe, has apparently not been studied with respect to the germination of its spores and this dearth of information directly prompted the present investigation. The work reported here is in two sections: (1) the determination of the optimum conditions for germination; (2) the elucidation of the compounds specifically responsible for breaking of dormancy.

\section{METHODS}

Organism. Clostridium bifermentans cN 1617 (Wellcome Research Laboratories Culture Collection Catalogue number) was used throughout. This organism has been clearly differentiated from the closely related pathogenic species $C$. sordellii by biochemical (Brooks \& Epps, 1958) and immunological methods (Walker, 1963).

Preparation of spore suspensions. The contents of an ampoule of dried culture were suspended in $20 \mathrm{ml}$. Brewer's thioglycollate (mercaptoacetate) medium (Brewer, 1940) and incubated at $37^{\circ}$. After $18 \mathrm{hr}$, subcultures (1 ml.) were transferred to 
nutrient broth containing $\mathbf{0 \cdot 2 5} \%$ glucose, and incubated at $37^{\circ}$ under anaerobic conditions for $72 \mathrm{hr}$. The resulting growth, consisting of 85-90\% phase-bright spores, was harvested by centrifugation and washed with sterile $0 \cdot 1$ m-phosphate

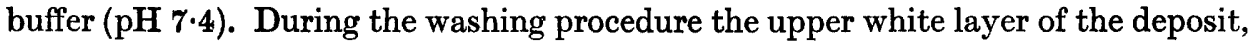
consisting largely of cellular debris and vegetative organisms, was suspended in the buffer solution and discarded (Long \& Williams, 1958). After washing five times with buffer, the suspension was aerated by vigorous shaking and stored overnight at $4^{\circ}$ to allow any remaining vegetative forms to lyse. The spores were then washed five more times with phosphate buffer and the volume of the final suspension adjusted to have an extinction value of $0.35-0.4$ at $680 \mathrm{~m} \mu(15 \mathrm{~mm}$. diam. tube; Hilger 810 Biochem. Absorptiometer), corresponding to approximately $10^{7}$ spores/ $\mathrm{ml}$.

Media. A medium (CMB) based on that described by Smith \& Douglas (1950) was used and had the following composition: acid-hydrolysed casein, $2 \%(w / v$; Oxoid, London); $\mathrm{KH}_{2} \mathrm{PO}_{4}, \mathbf{0 . 5} \%$ (w/v); biotin, nicotinamide, pyridoxal and calcium pantothenate, $10 \mu \mathrm{g} . / \mathrm{ml}$. each; $\mathrm{MgSO}_{4} .7 \mathrm{H}_{2} \mathrm{O}, 1 \cdot 6 \mathrm{mg} . / \mathrm{ml}$.; $\mathrm{MnSO}_{4} .4 \mathrm{H}_{2} \mathrm{O}, 80 \mu \mathrm{g} . /$ ml.; $\mathrm{FeSO}_{4} \cdot 7 \mathrm{H}_{2} \mathrm{O}, 80 \mu \mathrm{g} . / \mathrm{ml}$. The medium was adjusted to $\mathrm{pH} 8 \cdot 0$, boiled, filtered, readjusted to $\mathrm{pH} 7 \cdot 4$, and sterilized by autoclaving at $121^{\circ}$ for $20 \mathrm{~min}$.

Preparation of vitamin-free acid hydrolysed casein. This was prepared in order to study a possible vitamin requirement for the germination of spores. Commercial casein (British Drug Houses Ltd., vitamin- and fat-free) was extracted with methanol and hydrolysed by the method described by Barton-Wright (1961), by using litharge to decrease the concentration of chloride in the final product. The final solution was freeze-dried and solutions of this hydrolysate were prepared as detailed above, with or without the salts of magnesium, manganese and iron.

Yeast extract. A $25 \%$ solution (YE) was prepared by the method of Phillips \& Gibbs (1961).

Amino acids. For investigations of the amino acid requirements for germination of spores of Clostridium bifermentans, aqueous solutions of amino acids $(5 \mathrm{mg} . / \mathrm{ml}$; L. Light and Co.) were pasteurized by heating at $100^{\circ}$ for $20 \mathrm{~min}$., and stored at $4^{\circ}$ until required for use; $0.5 \mathrm{ml}$. volumes of amino-acid solutions were added to $5 \mathrm{ml}$. spore suspension.

Carboxylic acids. For work on the specific stimulants of germination of spores of Clostridium bifermentans aqueous solutions of the following carboxylic acids were used: lactic acid $\left(0.5 \%\right.$, v/v; B.P., $\left.\mathrm{C}_{3} \mathrm{H}_{6} \mathrm{O} 89.3 \%\right)$; oxalosuccinic acid Ba salt, DLisocitric acid lactone, cis-aconitic acid anhydride, oxaloacetic acid, $\alpha$-oxoglutaric acid and L-malic acid (from L. Light and Co.); succinic acid, citric acid (British Drug Houses Ltd., Analar). Aqueous solutions of these acids (5 mg./ml.) were stored at $4^{\circ}$.

Heat shock of spore suspensions. Samples $(5 \mathrm{ml}$.) of spore suspensions in sterile plugged tubes $(150 \times 15 \mathrm{~mm}$.) were heated in water baths at selected temperatures $\left( \pm 1^{\circ}\right)$ for $10 \mathrm{~min}$., after which the tubes were cooled rapidly in chilled water.

Adjustment of $\mathrm{pH}$ value of spore suspensions. Amounts of acid or alkali to adjust spore suspension to required $\mathrm{pH}$ values were calculated by reference to a titration curve of the $0 \cdot 1 \mathrm{M}$-phosphate buffer in which the spores were suspended.

Estimation of degree of germination. The germination of a spore suspension was followed by observing the decrease in extinction at $680 \mathrm{~m} \mu$. Samples of CMB 
medium (1 ml.) or of YE solution $(0.5 \mathrm{ml}$.) or both were added to $5 \mathrm{ml}$. of spore suspension, and measurements of extinction made at intervals of $10 \mathrm{~min}$. (Hilger 810 Biochem. Absorptiometer; Powell, 1950). The extent of germination of a spore suspension was also determined by observation of films stained with $1 \%$ aqueous methylene blue for $30 \mathrm{~min}$. (Powell, 1950; Levinson \& Sevag, 1953) and by phasecontrast microscopy (Pulvertaft \& Haynes, 1951). Those spores which were completely stained blue or were completely phase-dark were counted as having germinated.

\section{RESULTS}

Smith \& Douglas (1950) described the growth requirements of Clostridium bifermentans in a medium based on acid-hydrolysed casein. Since these workers used a washed spore suspension for inoculation, the medium they described was apparently sufficient to stimulate germination and to support vegetative growth and was therefore used in the investigations of the optimum conditions for the germination of spores of $C$. bifermentans.

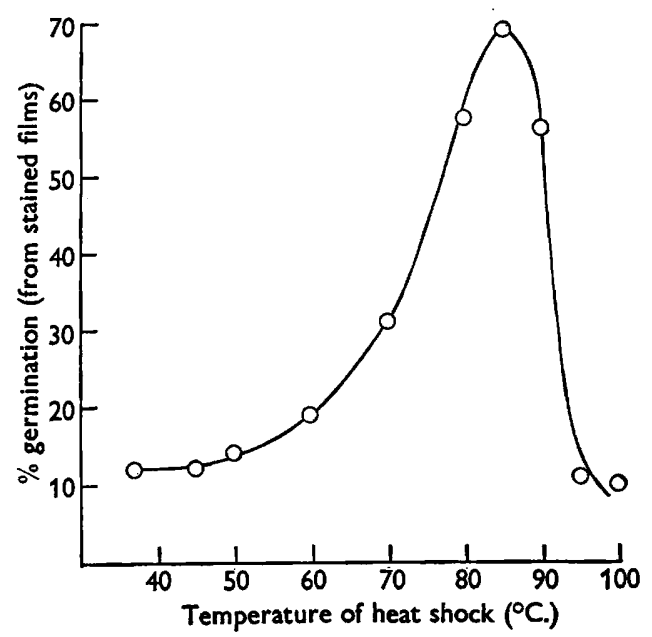

Fig. 1. The effect of temperature of heat shock for $10 \mathrm{~min}$. on the germination of spores of Clostridium bifermentans in CMB medium + yeast extract.

Effect of glucose, tryptophan, cystine, thioglycollate and yeast extract on germination. Though glucose, tryptophan, cystine and thioglycollate are essential for vegetative growth, they were not required for germination in medium based on acid-hydrolysates of casein. Indeed the presence of thioglycollate, in concentrations between 0.005 and $0.1 \%(v / v)$, markedly decreased the rate of germination as compared with that observed in the absence of thioglycollate. However, outgrowth and formation of a vegetative culture of Clostridium bifermentans occurred only when thioglycollate was present. The addition of yeast extract to acid-hydrolysates of casein increased the rate of spore germination.

Effect of heat shock on spore germination. Maximum germination in CMB medium + yeast extract at $37^{\circ}$ was attained following a heat shock for $10 \mathrm{~min}$. at $80-85^{\circ}$ (Fig. 1). Little germination of a spore suspension occurred when heated at $70^{\circ}$ or below, and temperatures of $90^{\circ}$ and above prevented subsequent germination. Spores heated at $95^{\circ}$ for $10 \mathrm{~min}$. did not lose phase-brightness but did take up 
methylene blue in the spore coats to a limited extent. Viable counts in agar media showed that $95^{\circ}$ for $10 \mathrm{~min}$. was lethal for $99.99 \%$ of the spores. In all subsequent work, spores were heated at $85^{\circ}$ for $10 \mathrm{~min}$., these conditions of heat shock being optimal for subsequent germination.

Effect of $p H$ value on germination. There appeared to be no sharp optimal $\mathrm{pH}$ value for germination of heat shocked spores in CMB medium + yeast extract

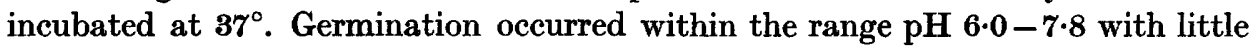
difference in rate. At $\mathrm{pH} \mathbf{5 . 0}$ and $\mathrm{pH} 8.5$ no germination occurred. Incubation at $\mathrm{pH} 10 \cdot 0$ or $\mathrm{pH} 3 \cdot 0$ had little effect on the ability of spores to germinate on subsequent re-adjustment to $\mathrm{pH} \mathbf{7 \cdot 4}$.
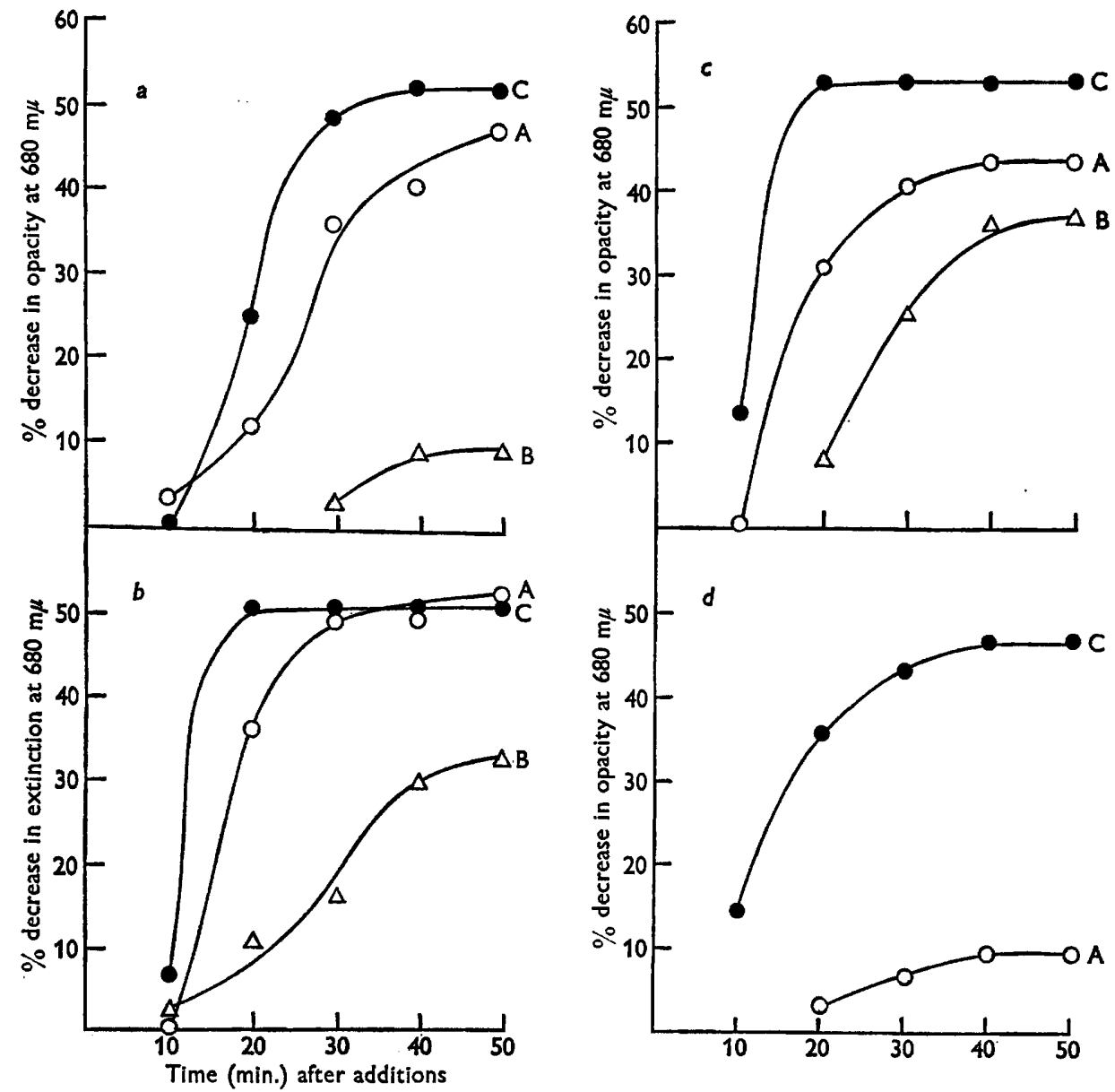

Fig. 2. The effect of temperature of incubation on the germination of suspensions of spores of Clostridium bifermentans. $a, 25^{\circ}: b, 30^{\circ}: c, 37^{\circ}: d, 45^{\circ}$. A, Omedium: $\mathbf{B}, \triangle \longrightarrow \triangle$, + yeast-extract: $\mathbf{C},-\longrightarrow$, $+\mathbf{C M B}+$ yeast extract. \% decrease in opacity $=\left(E_{0}-E_{x} / E_{0}\right) \times 100$ where $E_{0}$ and $E_{x}$ are extinctions immediately after the addition of germinating agents and at time $x$ respectively.

Effect of temperature of incubation. The germination of spores of Clostridium bifermentans in CMB and yeast extract both separately and as a mixture was investigated between 12 and $45^{\circ}$. At temperatures below $20^{\circ}$, little or no germination 
occurred with $60 \mathrm{~min}$. The maximum rate of germination at each temperature was attained in CMB medium + yeast extract (Fig. 2). At $45^{\circ}$ germination was markedly decreased, particularly in CMB medium and was absent in yeast extract. The optimum temperature of incubation was $37^{\circ}$ and all subsequent work was therefore carried out at this temperature.

\section{Investigation of the compounds specifically responsible for the germination of spores of Clostridium bifermentans}

Effect of vitamin-and fat-free casein hydrolysate. Medium prepared with vitaminand fat-free casein hydrolysate did not stimulate the germination of spores, whereas commercial casein hydrolysate did. Addition of yeast extract to the vitamin-free casein hydrolysate stimulated germination to a degree comparable with that obtained with CMB medium + yeast extract. The addition of the four vitamins biotin, nicotinamide, pyridoxal and pantothenate, salts of manganese, magnesium and iron to the vitamin-free hydrolysate had no effect. Amino acid analysis (Beckman-Spinco, Model 120 Amino Acid Analyzer) of the commercial and vitaminfree casein hydrolysates revealed no major differences in amino acid composition and addition of the commonly occurring L-amino acids to the vitamin-free casein hydrolysate did not stimulate germination in the absence of yeast extract. It seemed likely therefore that amino acids alone could not stimulate the germination of spores of Clostridium bifermentans, and that an unknown factor present in yeast extract was required.

Amino acid requirement for germination in the presence of yeast extract. Twentyseven L-amino acids were examined for their effect on germination in the presence of yeast extract. Of these L-phenylalanine, L-leucine and L-cystine markedly increased the rate of germination; glycine, L-valine, L-methionine and L-cysteine inhibited germination; the other amino acids tested were without effect (Fig. 3).

Replacement of yeast extract in the presence of vitamin-free casein hydrolysate. Since nucleosides have been implicated in the germination process of spores of Bacillus spp. (see Halvorson \& Church, 1957), the effect of some purine and pyrimidine bases and ribosides as replacements for yeast extract was examined. The addition of thymine, uracil, uric acid, guanine, adenine, xanthine, hypoxanthine, adenosine and guanosine did not stimulate spore germination in vitamin-free casein hydrolysate. Ultraviolet absorption spectra of solutions of commercial and vitamin-free casein hydrolysates showed no significant differences at $260 \mathrm{~m} \mu$, the region in which many purines and pyrimidines and their derivatives are strongly absorbing.

The other group of compounds of metabolic importance investigated included glucose and some of its degradation products, namely the carboxylic acids pyruvic, lactic, acetic, citric, cis-aconitic, isocitric, oxaloacetic, malic, succinic, $\alpha$-oxoglutaric and oxalosuccinic acids. In the presence of commercial casein hydrolysate, lactate, pyruvate, malate, succinate and oxaloacetate stimulated germination, but in vitamin-free casein hydrolysate only lactate had a marked effect on germination, and pyruvate and oxaloacetate a slight effect; succinate and malate were inactive. In a mixture of amino acids which simulated vitamin-free casein hydrolysate, lactate stimulated rapid germination, but pyruvate and oxaloacetate were very much less active. 
The specific stimulants of germination of spores of Clostridium bifermentans

Since germination of spores was obtained in a mixture of amino acids + lactate, an examination of those specific amino acids which were needed for the breaking of dormancy in the presence of lactate was made. A mixture of L-phenylalanine, L-leucine and L-cystine in the presence of lactate did not stimulate germination, but on addition of $L-\alpha$-alanine to this mixture, rapid germination occurred; $L$ - $\alpha$-alanine was not replaced by any other amino acid tested. Further investigations showed that $\mathrm{L}$-phenylalanine $+\mathrm{L}$ - $\alpha$-alanine + lactate constituted the specific requirements for germination and that L-phenylalanine could be replaced by L-leucine, although the rate of germination was then decreased (Fig. 4).

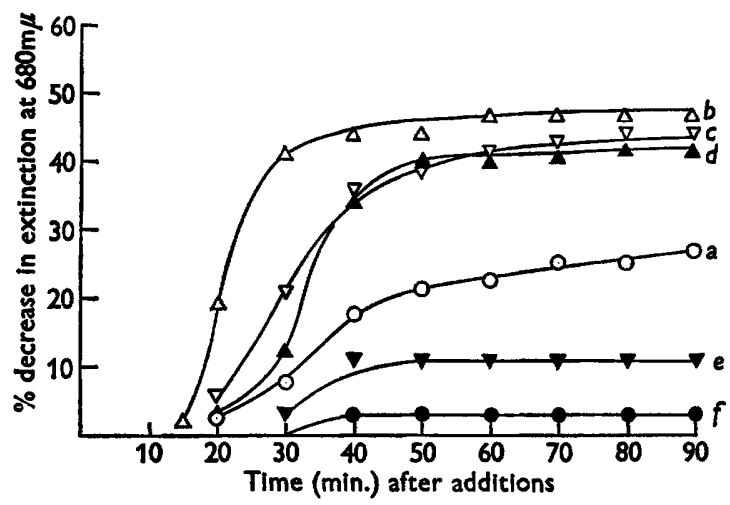

Fig. 3

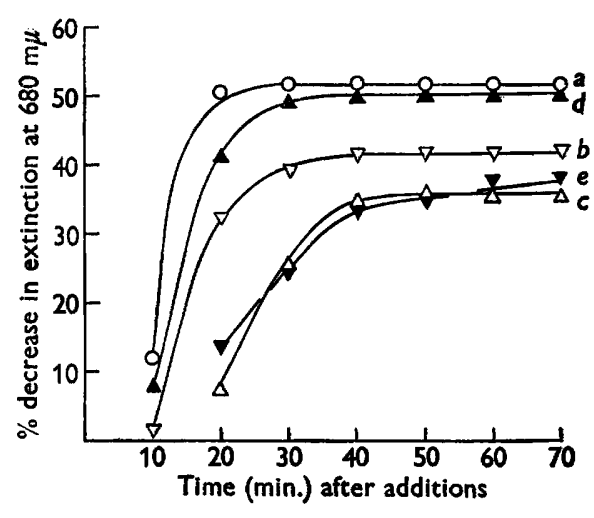

Fig. 4

Fig. 8. The effect of amino acids on the germination of suspensions of spores of Clostridium bifermentans in yeast extract (YE) solution. $a, \mathrm{O}-\mathrm{O},+\mathrm{YE}$ only: $b, \triangle-\triangle$, +YE + L-phenylalanine (50 $\mu \mathrm{g} . / \mathrm{ml}$.): $\quad c, \nabla-\nabla, \quad+\mathrm{YE}+\mathrm{L}-\mathrm{leucine}$ (50 $\mu \mathrm{g} . / \mathrm{ml}$.): $d, \Delta-\Delta,+\mathrm{YE}+\mathrm{L}$-cystine $(50 \mu \mathrm{g} . / \mathrm{ml}):. e, \nabla-\nabla,+\mathrm{YE}+\mathrm{L}$-methionine $(50 \mu \mathrm{g} . /$ ml.): $f,-1,+\mathrm{YE}+$ glycine, or L-valine or L-cysteine $(50 \mu \mathrm{g} . / \mathrm{ml}$. $)$.

Fig. 4. The minimum requirements for the germination of suspensions of spores of Clostridium bifermentans. $a, 0-0,+\mathrm{CMB}$ medium + yeast extract: $b, \nabla-\nabla$, + CMB medium: $c, \Delta-\Delta$, + yeast extract: $d, \Delta-\Delta,+\mathrm{I}-$ phenylalanine $(50 \mu \mathrm{g} . /$ $\mathrm{ml}.)+\mathrm{L}-\alpha$-alanine $(50 \mu \mathrm{g} . / \mathrm{ml})+$. lactic acid $(0.5 \mu \mathrm{l} . / \mathrm{ml}):. e, \nabla-\nabla,+\mathrm{L}$-leucine $(50 \mu \mathrm{g} . /$ ml.) + L- $\alpha$-alanine $(50 \mu \mathrm{g} . / \mathrm{ml}$.) +lactic acid $(0.5 \mu \mathrm{l} . / \mathrm{ml}$. $)$.

\section{DISCUSSION}

Although the heat resistance of spores is usually regarded as the feature that most clearly differentiates them from the vegetative forms, spores may be clearly recognized by other properties. Thus, the heat sensitivity of spores of some aerobic and anaerobic bacilli is paralleled by loss of phase-brightness, increased permeability to stains and decrease in turbidity of spore suspensions, so making it possible to use the latter group of properties as criteria of germination. The criteria of germination used in the present work were the microscopic appearance and change in turbidity of suspensions of spores. In common with spores of the aerobic bacilli (Powell \& Hunter, 1955) spores of Clostridium bifermentans were found to require activation by heat shock before rapid and complete germination ensued. This observation appears to distinguish the spores of $C$. bifermentans from those of $C$. roseum studied by Hitzman, Halvorson \& Ukita (1957). 
Although strictly anaerobic conditions are required for the vegetative growth of Clostridium bifermentans, these conditions are not essential for the germination of its spores. Indeed the addition of thioglycollate markedly inhibited germination as previously reported for spores of $C$. botulinum (Treadwell, Jann \& Salle, 1958). The germination of spores of other species of the genus Clostridium is apparently not inhibited by thioglycollate, and anaerobic conditions are apparently required for germination to occur (Wynne, Mehl \& Schmieding, 1954; Hitzman et al. 1957). By making experiments under aerobic conditions therefore, it was possible in the present work to differentiate between germination and subsequent outgrowth since the latter (see above) will only take place under anaerobic conditions. Thus it was possible to observe separately the three major phases in the transition from dormant spores to vegetative forms, namely activation, germination and outgrowth.

The compounds found specifically responsible for stimulating the germination of spores of Clostridium bifermentans included $\mathrm{L}$ - $\alpha$-alanine and L-phenylalanine, compounds also required by spores of $C$. roseum (Hitzman et al. 1957). L- $\alpha$-Alanine is also required by the spores of many aerobic bacilli (Thorley \& Wolf, 1960), and it is possible that $\mathrm{L}$ - $\alpha$-alanine is essential for the germination of spores of aerobic and anaerobic bacilli. However, lactic acid has apparently not been reported previously to be required for the germination of spores, although some dicarboxylic acids have been reported to stimulate the germination of spores of $C$. botulinum (Wynne \& Foster, 1948).

The author wishes to thank Miss B. M. Hill for technical assistance and Dr A. W. Phillips for help in preparing the manuscript.

\section{REFERENCES}

Barton-Wright, E. C. (1961). Practical methods for the microbiological assay of the vitamin $B$ complex and amino acids. Lab. Practice, 10, 715.

Brewer, J. H. (1940). Clear liquid mediums for the aerobic cultivation of anaerobes. J. Amer. Med. Ass. 115, 598.

Brooks, M. E. \& EPPs, H. B. G. (1958). Taxonomic studies of the genus Clostridium: Clostridium bifermentans and C. sordellii. J. gen. Microbiol. 21, 144.

Halvorson, H. O. \& Church, B. (1957). Biochemistry of spores of aerobic bacilli with special reference to germination. Bact. Rev. 21, 112.

Hitzman, D. O., Halvorson, H. O. \& Ukita, T. (1957). Requirements for production and germination of spores of anaerobic bacteria. J. Bact. 74, 1.

Levinson, H. S. \& Sevag, M. G. (1953). Stimulation of germination and respiration of spores of Bacillus megatherium by manganese and monovalent anions. J. gen. Physiol. 36, 617 .

Long, S. K. \& Williams, O. B. (1958). A method for removal of vegetative cells from bacterial spore preparations. J. Bact. 76, 332.

Phillirs, A. W. \& Gibis, P. A. (1961). Techniques for the fractionation of microbiologically active peptides derived from casein. Biochem.J. 81, 551 .

Poweld, J. F. (1950). Factors affecting the germination of thick suspensions of Bacillus subtilis spores in L-alanine solution. J. gen. Microbiol. 4, 330.

Powell, J. F. \& HunTER, J. R. (1955). Spore germination in the genus Bacillus: the modification of germination requirements as a result of preheating. J. gen. Microbiol. 13, 59.

Pulvertaft, R. J. V. \& Haynes, J. A. (1951). Adenosine and spore germination; phasecontrast studies. J.gen. Microbiol. 5, 657. 
Smith, L. DE S. \& Dougras, H. C. (1950). Factors necessary for maximum growth of Clostridium bifermentans. J. Bact. 60, 9.

Thorley, C. M. \& Wolf, J. (1960). Some Germination Factors of Mesophilic Spore Formers, Spores II (1961). Ed. H. O. Halvorson. Minneapolis 15, Minn.: Burgess Publishing Co. Treadweld, P. E., Jann, G. J. \& Salue, A. J. (1958). Studies on factors affecting the rapid germination of spores of Clostridium botulinum. J. Bact. 76, 549.

WALKER, P. D. (1963). The spore antigens of Clostridium sporogenes, Cl. bifermentans and Cl. sordellii. J. Path. Bact. 85, 41.

Wynne, E. S. \& Foster, J. W. (1948). Physiological studies on spore germination with special reference to Clostridium botulinum. 3. Carbon dioxide and germination, with a note on $\mathrm{CO}_{2}$ and aerobic spores. J. Bact. 55, 331.

Wynne, E. S., Meht, D. A. \& Schmieding, W. R. (1954). Germination of Clostridium spores in buffered glucose. $J$. Bact. 67, 435 . 